\title{
Editorial: Subjective Perceptions of Memory Functioning in Old Age - Nature, Correlates, and Developmental Trajectories
}

\author{
Gizem Hülür Denis Gerstorf \\ Institute of Psychology, Humboldt University, Berlin, Germany
}

\author{
Key Words \\ Cognitive aging $\cdot$ Subjective memory $\cdot$ Memory \\ performance
}

\begin{abstract}
Subjective memory complaints are often used as diagnostic criteria for several neurocognitive disorders. Although a number of studies have examined subjective memory and its associations with memory functioning in adulthood and old age, it is still an open question whether subjective perceptions of one's memory indicate actual memory functioning or whether they are rather derived from factors other than memory, such as depressive symptoms. The studies in this special section examine subjective perceptions of memory functioning and their associations with objectively measured memory performance in general and in clinical populations. The four articles adopt cross-sectional and longitudinal methodologies and offer key insights into the nature, correlates, and developmental trajectories of subjective memory. To begin with, the studies compiled in this special section demonstrate that changes in subjective memory perceptions are indeed associated with changes in memory performance [Zimprich and Kurtz, this issue, pp. 223-231], but the size of associations between levels of and changes in subjective memory and memory performance is in part modulated by personality characteristics and depressive symptoms [Hülür et al., this issue, pp. 232-240]. Second, the studies compiled
\end{abstract}

here show that factors other than memory are also closely associated with memory perceptions, including functional health as well as domain-general and health-specific control beliefs [Luszcz et al., this issue, pp. 241-250]. Third, the study by Thompson et al. [this issue, pp. 251-257] shows that selfand informant-reports of retrospective and prospective memory difficulties are not associated with performancebased measures and does not sufficiently differentiate between healthy controls and patients diagnosed with mild cognitive impairment or dementia. In our editorial, we put these findings in perspective and discuss implications for research and practice. To extend our knowledge, we conclude by outlining two key avenues for future research: (i) longitudinal multivariate studies of the construct space surrounding subjective memory and (ii) the viability of experience sampling studies with daily or hourly measurements to tackle some of the mechanisms underlying these associations.

(c) 2015 S. Karger AG, Basel

\section{Introduction}

Complaints about declining memory are common among older adults [1]. Due to recent demographic changes towards an aging population, it is becoming increasingly important to understand the nature and correlates of subjective perceptions of memory functioning and whether memory complaints actually signify memo-

\section{KARGER 125}

(c) 2015 S. Karger AG, Basel

0304-324X/15/0613-0218\$39.50/0

E-Mail karger@karger.com

www.karger.com/ger
Gizem Hülür

Institute of Psychology, Humboldt University Berlin

Unter den Linden 6

DE-10099 Berlin (Germany)

E-Mail gizem.hueluer@hu-berlin.de 
ry impairment. The four studies included in this special section use cross-sectional and longitudinal data to examine how subjective memory and objectively measured memory performance are related in general and in clinical populations. Furthermore, the articles examine the role of individual difference variables other than memory, including depressive symptoms, functional health, control beliefs, personality, and subjective age. In this editorial, we will first present some of the underlying themes and rationales. Next, we will highlight important findings from the articles included in this special section. Finally, we will outline two avenues for future research that promise to help us better understand potential underlying mechanisms: (i) longitudinal multivariate studies of the construct space surrounding subjective memory and (ii) the viability of experience sampling studies.

\section{Background}

To understand people's evaluations of their own memory functioning, it is important to consider the multitude of factors that can have an influence on such perceptions and evaluations. What goes on within an individual when they evaluate their memory functioning? Individuals might engage in social comparisons with others [1]; however, their reference points are often unclear. It is highly unlikely that people compare themselves with a representative group of similar-aged individuals on actual memory functioning. In line with this reasoning, several crosssectional studies have found only weak associations between subjective memory and memory performance as shown in a recent meta-analytic study [2].

Another line of reasoning suggests that individuals' ratings are based on their experiences of memory failures and successes over time $[1,3]$. Thus, when people experience steep declines of memory performance over time, their perceptions of memory functioning should also decline over this time period. This notion suggests that changes in subjective memory and changes in memory performance should be correlated. While some longitudinal studies find correlated change between subjective memory and memory performance [4], others do not [5]. The reasons for this discrepancy are not yet clear. The studies differ in a number of methodological aspects, such as strategies for participant recruitment, age ranges (middle-aged and older participants [4]; only older participants [5]), and different operational definitions of subjective memory (single-item rating [4]; multi-item scale measuring memory complaints [5]). Taken together, it is still an open question whether and how changes in subjective memory and memory performance are associated. This special section includes two studies that have examined associations between subjective memory and memory performance over time $[6,7$, this issue].

Individuals may make use of other information when rating their memory functioning. For example, on a day when people experience a dysphoric mood, their ratings of memory functioning may be more negative. In line with this reasoning, previous research has found that depressive symptoms were associated with subjective memory even after controlling for actual memory performance $[4,5]$. Aside from depressive symptoms, a number of other individual difference characteristics have been related to subjective memory, such as neuroticism [5] or functional health [4]. Also, individuals' memory ratings might be based on their implicit theories about age-related memory declines [3]. An individual who expects age-related declines in memory might rate his or her memory functioning less favorably as compared to an individual who believes that memory functioning can be maintained into old age. The special section includes two studies that have examined correlates of subjective memory and memory performance, including personality, subjective age [7, this issue], functional health, as well as domain-general and health-specific control beliefs [8, this issue].

So far, subjective memory complaints are often used as diagnostic criteria for neurocognitive disorders (e.g. mild cognitive impairment, $\mathrm{MCI}$ ). One concern about the use of subjective measures is related to anosognosia, i.e. that individuals with a cognitive impairment might lack insight into their own condition. In line with this reasoning, many studies showed that informant ratings typically evince stronger associations with cognitive performance [9]. It is still an open question whether informant ratings are more valid than self-ratings for different memory subtypes (e.g. prospective memory). The paper by Thompson et al. [10, this issue] examines self-reports and informant reports of retrospective and prospective memory and their associations with cognitive measures in cognitively healthy and clinical samples.

\section{The Special Section}

This special section brings together a collection of articles evaluating the nature, correlates, and developmental trajectories of subjective perceptions of memory functioning. All studies thoroughly examine different aspects of the viability of subjective perceptions in the study of 
Table 1. Overview of design characteristics and findings in each of the four studies

\begin{tabular}{|c|c|c|c|c|}
\hline & Zimprich and Kurtz & Hülür et al. & Luszcz et al. & Thompson et al. \\
\hline \multicolumn{5}{|l|}{ Sample } \\
\hline Subjects, $\mathrm{n}$ & 236 & 15,824 & 2,087 & 138 \\
\hline Female gender, $\%$ & 45 & 58 & 49 & 51 \\
\hline Population type & $\begin{array}{l}\text { ZULU participants, } \\
\text { residents of Zurich }\end{array}$ & $\begin{array}{l}\text { HRS participants, } \\
\text { national US sample }\end{array}$ & $\begin{array}{l}\text { ALSA participants, } \\
\text { residents of South } \\
\text { Australia }\end{array}$ & $\begin{array}{l}53 \text { cognitively healthy } \\
\text { participants, } 48 \text { participants } \\
\text { with MCI, } 37 \text { participants } \\
\text { with dementia }\end{array}$ \\
\hline Measure of subjective memory & $\begin{array}{l}\text { Two subscales from the } \\
\text { abridged MIA } \\
\text { questionnaire: memory } \\
\text { capacity and change }\end{array}$ & $\begin{array}{l}\text { Single item } \\
\text { 'How would you rate } \\
\text { your current memory?' } \\
0 \text { (poor) to } 4 \text { (excellent) }\end{array}$ & $\begin{array}{l}\text { Two subscales from } \\
\text { the MIA } \\
\text { questionnaire: locus } \\
\text { and task }\end{array}$ & $\begin{array}{l}\text { PRMQ, self-report and } \\
\text { informant report }\end{array}$ \\
\hline Measurement occasions, $\mathrm{n}$ & 2 & Up to 4 & Up to 4 & 1 \\
\hline Time span, years & 5 & 6 & 12 & - \\
\hline Change-change & $\begin{array}{l}\text { Capacity: } r=0.54^{*} \\
\text { Change: } r=-0.44^{*}\end{array}$ & $\mathrm{r}=0.49^{*}$ & $\begin{array}{l}\text { Locus: no variance } \\
\text { in change } \\
\text { Task: } \mathrm{r}=-0.35^{*}\end{array}$ & - \\
\hline
\end{tabular}

Values in parentheses represent ranges. ZULU = Zurich Longitudinal Study on Cognitive Ageing; HRS = Health and Retirement Study; ALSA = Australian Longitudinal Study of Ageing; MIA = Metamemory in Adulthood [14]; PRMQ = Participant Self-Report Questionnaire [15]. * $\mathrm{p}<0.05$.

cognitive aging and highlight theoretical and practical implications. We first summarize each article, and then provide some overall inferences that can be drawn from this collection of articles. The design characteristics of the four studies are summarized in table 1, which also shows the associations between levels of and changes in subjective memory and memory performance reported in each of the four studies.

The first article by Zimprich and Kurtz [6, this issue] used two-wave longitudinal data obtained across 5 years from the Zurich Longitudinal Study on Cognitive Ageing and identified correlated changes between subjective memory and memory performance that were stronger than associations found at baseline assessment. The findings were consistent across the two measures of subjective memory examined, i.e., subjective assessments of memory capacity and memory change. The results suggested robust associations between changes in subjective memory and changes in memory performance $(r=0.54$ for memory capacity, and $\mathrm{r}=-0.44$ for memory change), but correlations were not strong enough to be able to recommend substituting objective tests with subjective assessments.

The second article by Hülür et al. [7, this issue] used 6-year longitudinal data from the Health and Retirement Study and also showed correlated changes between subjective memory and memory performance. The strengths of associations, however, varied by personality (conscientiousness and agreeableness) and depressive symptoms, suggesting that subjective memory complaints can arise from multiple sources and association between (changes in) subjective memory and (changes in) memory performance might be stronger among certain segments of the population than among others.

The third article by Luszcz et al. [8, this issue] used 12year longitudinal data from the Australian Longitudinal Study of Ageing and identified longitudinal associations between memory, functional health, and subjective control beliefs in multiple domains, highlighting the interrelatedness of these constructs. Luszcz et al. examined tra- 
jectories of two different aspects of subjective memory beliefs, with knowledge of the memory system being stable over time and perceived control over memory increasing, which suggests that individuals attribute memory failures and successes more and more to themselves rather than to external circumstances. Their findings suggest that knowledge of the memory system is another key factor to be taken into account when examining subjective memory complaints in older adults.

The fourth article by Thompson et al. [10, this issue] evaluated the validity of self-ratings and informant ratings of prospective memory in clinical populations by linking these with performance measures of prospective memory and general cognition in three groups (patients with MCI, patients with dementia, and healthy controls). Findings suggest that self- and informant-reported prospective memory is not viable to distinguish between healthy controls and clinical groups and may not be sensitive enough to detect deficits of prospective memory functioning.

The articles included in this special issue shed light on various aspects of the nature and correlates of subjective memory (and its change across adulthood and old age). All studies examined how subjective perceptions correspond to performance in cognitive tests, and the first three articles examined such associations longitudinally. Although subjective assessments of memory function cannot substitute a thorough assessment with objective diagnostic instruments, the collection of articles in the special section suggests that they relate to memory performance in longitudinal data that span across longer time frames of multiple years. Also, the special section highlighted a multitude of other factors associated with subjective perceptions of memory functioning. Finally, the utility of self-reports and informant reports of memory complaints in clinical settings was called into question as they cannot sufficiently predict memory deficits.

\section{Future Directions}

Finally, we will highlight two avenues for future research to address some of the open questions identified above. Previous research has suggested that the etiology of subjective perceptions of memory functioning is complex. Future research should thus examine how these factors contribute to subjective memory. Also, experience sampling studies might help us better understand how the mechanisms behind memory complaints evolve and manifest in people's lives.

Subjective Memory in Old Age

\section{Multivariate Studies of the Construct Space}

Longitudinal multivariate studies of the construct space around subjective memory would allow evaluating its convergent and discriminant validity. If individuals are able to monitor their memory functioning accurately, subjective memory should show convergent validity for memory performance, i.e., changes in subjective memory and memory performance should be correlated. The convergent validity of subjective memory has been examined in many studies [4-7]. Studies of discriminant validity examine whether subjective memory is more closely related to memory performance as compared to other constructs. For example, in a recent study [4], changes in subjective memory were correlated both with changes in memory performance and depressive symptoms. It is possible that sub-facets of depressive symptoms show differential associations with subjective memory. For example, among the neuroticism sub-facets, self-consciousness was the strongest predictor of subjective memory complaints, suggesting that individuals prone to feeling embarrassed view their memory functioning less favorably [11], possibly because they perceive memory failures in social settings more negatively than others [3]. Based on this reasoning, it could be expected, for example, that interpersonal symptoms of depression, such as feelings of inferiority, should show the strongest associations with subjective memory.

Health could be another possible source of subjective memory complaints. Individuals could generalize their health complaints to the cognitive domain as suggested by the finding of Luszcz et al. [8, this issue] that declines in functional health were associated with increases in selfreported memory knowledge. Age-related expectations or stereotypes could also play a role. For example, Hülür et al. [7, this issue] showed that average levels of subjective age predicted subjective memory over time. Currently, little is known about whether changes in subjective memory and subjective age are correlated. Future studies should seek to examine longitudinal multivariate associations of subjective memory to gain a better understanding of the construct space.

\section{Experience Sampling Studies}

So far, memory perceptions have typically been considered as a stable trait [3], and fluctuations have rarely been examined. A recent study [4] found systematic occasion-to-occasion fluctuations of subjective memory, memory performance, and depressive symptoms, suggesting that subjective memory fluctuates like many other psychological characteristics [for an overview of the 
promise and challenges of multi-time scale studies, see 12]. However, this study only examined biennial fluctuations. Studying fluctuations in memory perceptions on a moment-to-moment and day-to-day basis would allow for studying possible underlying mechanisms. For example, it may be argued that individuals experiencing depressive symptoms might have a negative cognitive bias [13], which may lead them to be more sensitive toward everyday fluctuations of memory performance [4]. The experience of a memory failure, such as forgetting an appointment, may thus be more detrimental to people who report more depressive symptoms. Also, the issue of anosognosia and informant ratings [9] could be addressed in a daily diary study. For example, it could be examined whether informants' ratings reflect the fluctu- ating memory performance of the individual with cognitive impairment more accurately than self-ratings. In summary, the study of subjective memory and related constructs in daily life allows for a more mechanism-oriented inquiry in healthy old adults and individuals with cognitive impairment.

Taken together, this special section offers novel insights into the nature and correlates of subjective memory in general and clinical populations and furthers our understanding of the associations of subjective memory with memory performance and other interrelated factors. In addition, the series of articles also identifies avenues for future research that extend our knowledge on how individuals perceive the nature and correlates of cognitive aging.

\section{References}

1 Perrig-Chiello P, Perrig WJ, Stahelin HB: Differential aspects of memory self-evaluation in old and very old people. Aging Ment Health 2000;4:130-135.

-2 Crumley JJ, Stetler CA, Horhota M: Examining the relationship between subjective and objective memory performance in older adults: a meta-analysis. Psychol Aging 2014; 29:250-263.

3 Hertzog C, Pearman AM: Memory complaints in adulthood and old age; in Perfect TJ, Lindsay DS (eds): Handbook of Applied Memory. London, Sage, 2014, pp 423-443.

-4 Hülür G, Hertzog C, Pearman A, Ram N, Gerstorf, D: Longitudinal associations of subjective memory with memory performance and depressive symptoms: between-person and within-person perspectives. Psychol Aging 2014;29:814-827.

5 Pearman A, Hertzog C, Gerstorf D: Little evidence for links between memory complaints and memory performance in very old age: longitudinal analyses from the Berlin Aging Study. Psychol Aging 2014;29:828-842.
6 Zimprich D, Kurtz T: Subjective and objective memory changes in old age across five years. Gerontology 2015;61:223-231.

-7 Hülür G, Hertzog C, Pearman AM, Gerstorf D: Correlates and moderators of change in subjective memory and memory performance: findings from the Health and Retirement Study. Gerontology 2015;61:232-240.

8 Luszcz MA, Anstey KJ, Ghisletta P: Subjective beliefs, memory and functional health: change and associations over 12 years in the Australian Longitudinal Study of Ageing. Gerontology 2015;61:241-250.

$\longrightarrow 9$ Farias S, Mungas D, Jagust W: Degree of discrepancy between self and other-reported everyday functioning by cognitive status: dementia, mild cognitive impairment, and healthy elders. Int J Geriatr Psychiatry 2005; 20:827-834.

10 Thompson CL, Henry JD, Rendell PG, Withall A, Brodaty $\mathrm{H}$ : How valid are subjective ratings of prospective memory in mild cognitive impairment and early dementia? Gerontology 2015;61:251-257.
Pearman A, Storandt M: Self-discipline and self-consciousness predict subjective memory in older adults. J Gerontol B Psychol Sci Soc Sci 2005;60B:P153-P157.

12 Gerstorf D, Hoppmann CA, Ram N: The promise and challenges of integrating multiple time-scales in adult developmental inquiry. Res Hum Dev 2014;11:75-90.

13 Crane MK, Bogner HR, Brown GK, Gallo JJ: The link between depressive symptoms, negative cognitive bias and memory complaints in older adults. Aging Ment Health 2007;11: 708-715.

14 Smith G, Della Sala S, Logie RH, Maylor EA: Prospective and retrospective memory in normal aging and dementia: a questionnaire study. Memory 2000;8:311-321.

15 Dixon RA, Hultsch DF, Hertzog C: The Metamemory in Adulthood (MIA) questionnaire. Psychopharmacol Bull 1988;24:671688. 